\title{
Lateral Offset Based Analysis of Traffic Features at Urban Bottlenecks
}

\author{
He Zhao-Cheng, Zhang Li-Cheng, Sun Wen-Bo \\ Research Center of Intelligent Transportation Systems, Sun Yat-Sen University, Guangzhou 510000, \\ China,hezhch@mail.sysu.edu.cn
}

\begin{abstract}
There are a lot of vehicles changing its lane unobservantly when passing a traffic bottleneck in China, which may affect other vehicles that drive according to the rules. Even the traffic features on the road may also be influenced. Based on the data that collected from the road, this paper firstly proposes that there are different lateral interferences among three lanes at the bus stop bottleneck, and then proves that significant velocity differences are existed among three adjacent lanes. Besides, as the lateral interference often occurs at the bottleneck, lateral parameters are added to help to analyze the traffic features of the urban bottleneck. Finally this paper confirms the hypothesis that velocity will decrease when lateral offsetis increasing under the same density level. The result also shows that though the increase of the road density is the main reason of low passing speed at the traffic bottleneck, the lateral position also influences the average velocity greatly.
\end{abstract}

Keywords-transportation engineering; density level; traffic bottleneck; lateral offset; average velocity

\section{Introduction}

Traffic congestion is the most focus of the urban transportation issues, and traffic congestion often occurs at traffic bottlenecks. If there is a congestion section, vehicles in other countries may wait in line to pass it, while many vehicles in China will probably change their lanes unobservantly to get a better position or opportunity to pass it, which often causes some bad phenomenon like two lanes contains three vehicles. On the one hand, this bad driving habit in China will increase the road density and decrease the traffic volume; on the other hand, it can reduce the traffic capacity and road service level extremely, as lane changing always goes with slamming brakes. Therefore, study the change of the macroscopic traffic flow at urban bottlenecks is very meaningful for analyzing how traffic congestion emerges.

Before changing to another lane, vehicles must make a lateral movement, which will cause a lateral offset. With the lateral movement of the lane changing vehicles, the following vehicles and vehicles in the adjacent lanes will also be forced to make a lateral movement sometimes. Generally, at a traffic bottleneck, vehicles won't make a lateral movement unless there are obstacles in front of them, for example low speed cars or something else. Considering their own security, most drivers would slow down before the lateral movement, meanwhile,the following vehicles and vehicles in the adjacent lanes would be forced to slow down as well. Therefore, the lateral movement behavior of the vehicles will decrease the average velocity of the traffic bottlenecks.

As the lateral movement always occurs at traffic bottlenecks, it is necessary to analyze the lateral features at urban bottlenecks to see whether lateral movement would jam the road.

There are already many researches on traffic bottlenecks. Some researchers use fundamental diagram of traffic flow to identify the traffic state at bottlenecks ${ }^{[1-3]}$, while some others use cumulative curve to do $\mathrm{so}^{[4-5]}$. But they all only focus on longitudinal traffic flow parameters like volume, density, velocity, occupancy etc.

Few researchers paid attention to the lateral parameters. A study has shown that except for a small account of road traffic, four parameters related to the bus stop, bus stopping time for example, would make a vehicle to cause a lateral movement ${ }^{[6]}$. But it just illustrated the behavior that following vehicles would swerve sharply to avoid the stopping bus, without proposing the concept on the lateral offset and studying it. A study of expressway confirmed that traffic flow at expressway bottlenecks have two-capacity phenomenon, and it proposed that currently volume-velocity curve would overestimate the capacity of expressway bottlenecks ${ }^{[7]}$. But it did not continue in-depth analyzingon the reason why the capacity would be overestimated.

In addition, some people did study on the lateral movement of vehicles. A literature claimed that the lateral movement of non-motorized vehicles would affect the headway of the vehicles running in the adjacent lane ${ }^{[8]}$, yet its study object was mixed traffic flow. A research had emulated drivers'speed, deceleration, and lateral position of vehicles at rural intersections [9]. Butit was just for traffic accident analysis. There was another issue reported that when added the lateral offset to the conventional car-following model, the traffic flow would remain a steady state though the headway decreased ${ }^{[10]}$. However, it just used microscopic traffic flow, instead of macroscopic traffic flow, to study the influence of the lateral offset to the bottlenecks.

There is always existed in the urban bottlenecksthat road density is not very high, while the average speed is low or even zero. This condition always goes with large amount of unobservantly lane changing. This paper surveyed the lateral position of vehicles that went through an urban traffic bottleneck for example, and studied whether there is a 
relationship between lateral position and velocity of vehicles when the road density is invariable. In the later section, a new method, which is based on the lateral offset of vehicles, will be proposed to analyze the speed at the bottlenecks from a macro aspect.

\section{Data Description}

\section{A. Data Collection}

The urban traffic bottleneck site used in this study is the segment of the Guangzhou Avenueillustrated in Fig.1.The bus stop is about $20 \mathrm{~m}$ long, and the shoulder lane is Bus Lane, whose width is only $1.5 \mathrm{~m}$. When a bus is going to stop at the bus stop, it makes the road a bottleneck. The bottleneck begins in section 1.As the Fig. 1 shows, the box area from lane 1 to lane 3 wasdefined as the survey area, which was to record the traffic parameters by each lane. All data in this paper were collected from the CCTV at the entrance of the box area. Parts of the data from the two-day data wererandomly selected. Road density, average velocity and lateral position were collected as the mainly features.

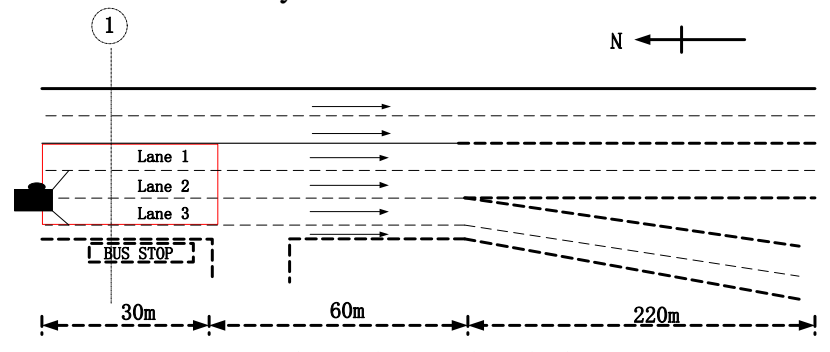

Fig.1 Urban Bottleneck Site

Because not all vehicles are driving along the centerline, and vehicles will interference others that are driving in the adjacent lane if they are driving too close to the lane dividers, so lateral position, which is accurate to the lane level to help to analyze the vehicle position, is defined. Taking every two adjacent center lines of the lane dividers as thedemarcations, each lane is divided into 18 equal parts. Taking the Fig. 1 for an example, each lane is numbered from east to west, and the position of each vehicle is where its central line is.

\section{B. Data Processing}

In order to facilitate the data analysis, road density is divided into 5classes as in Tab.1, where $\mathrm{k}=$ road density. Besides, this paper hold that the greatest lateral offset is the lateral offset of the sample. The vehicle which has the greatest lateral offset not only has the different driving behavior to other vehicles, but influent the following vehicles and vehicles on the adjacent lanes as well.

Table 1: Density classification

\begin{tabular}{cc}
\hline Density level & $\mathrm{k} \leq 50 \mathrm{pcu} / \mathrm{km}$ \\
\hline 1 & $50 \mathrm{pcu} / \mathrm{km}<\mathrm{k} \leq 83 \mathrm{pcu} / \mathrm{km}$ \\
2 & $83 \mathrm{pcu} / \mathrm{km}<\mathrm{k} \leq 117 \mathrm{pcu} / \mathrm{km}$ \\
3 & $117 \mathrm{pcu} / \mathrm{km}<\mathrm{k} \leq 150 \mathrm{pcu} / \mathrm{km}$ \\
4 & $150 \mathrm{pcu} / \mathrm{km}<\mathrm{k} \leq 183 \mathrm{pcu} / \mathrm{km}$ \\
\hline
\end{tabular}

Based on the lateral position, the lateral offset ( $\mathrm{L}$ for short) is defined. Suppose that the width of each lane is $l$. If the centerline of the vehicle coincide to the centerline of the lane when the vehicle going through the test section, the lateral offset is 0. Every time when the vehicle makes a lateral movement away from the centerline $18 /$ distance, the lateral offset will plus 1 , and the maximum of the lateral offset is 9 .

\section{Traffic Features on the Bottleneck}

\section{A. Average Velocity}

The road average speed can probably reflect a road traffic state.Fig. 2 shows the relationship between density level(D for short) and velocityin each lane. When D is less than 3, dispersion of the speed in each lane is very high, especially in lane 3 . But when the density level comes to a high level like 4 and 5, dispersion of the speed are not so high. As the highest speed in each lane at the same density level is almost the same, degree of dispersion of the speed in each lane is due to the lowest speed. When $\mathrm{D}=1$, for example, the highest speed in 3 lanes are about $16 \mathrm{~m} / \mathrm{s}$, while the lowest speed are about $9 \mathrm{~m} / \mathrm{s}$, $7 \mathrm{~m} / \mathrm{s}, 4 \mathrm{~m} / \mathrm{s}$. It shows that (1) though there being little vehicles on the road, the average speed may be low as well; (2) there may be some difference among the distribution of speed in each lane when the density level is low.

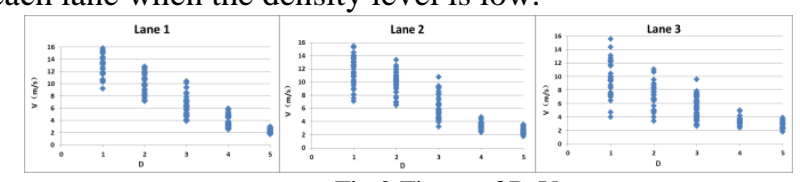

Fig.2 Figure of D-V

\section{B. Lateral Position}

Considering the basic situation of the road, when the density level is the same, there is one difference among the vehicles in each lane, the lateral position. Fig. 3 reveals the distribution of distance from the median edge, which reflects the lateral position directly. The distribution in lane 3 is more evenly than other lanes, so vehicles in lane 3 suffered the most severe lateral interference. Not only the lane changing of vehicles in lane 2 will interfere the normal driving in lane 3 , but the stopping buses near the bus stop will do it so. Especially, because the Bus Lane is just $1.5 \mathrm{~m}$ wide, when there is a bus stopping at the bus stop, vehicles in lane 3 have toacross the lane divider and almost change to lane 2 to pass the road. The distribution in lane 1 is the most concentrated of all, so vehicles in lane 1 suffered the least lateral interference. Because solid line is on the one side of lane 1, and both lane land lane 2 are go straight to the same downstream, there is little lateral interference at all. In addition, lane 2 also suffered the lateral interference sometimes. On the one hand, several vehicles change their path from lane 3 to lane 2 , and on the other hand, vehicles which have made a huge lateral offsetbecause of the stopping bus in lane 3 will also interfere the normal driving in lane 2. Yet both the lateral interferences are not force vehicles to make a great lateral offset.

Because the distribution of lateral position in lane 1 is 
normal, and it is similar to the distribution of ordinary sections, lane 1 can be considered as an ordinary section. Meanwhile, lane 3 is the shoulder lane that is near the bus stop. When it is a bus going to the bus stop, it will form a bottleneck near the bus stop, so lane 3 can represent a bottleneck section. The difference distribution of lateral position between lane 1 and lane 3 reveals that there is significant difference on the distribution of lateral position between ordinary section and bottleneck section. Therefore, it is necessary to analyze the lateral parameters at the bottlenecks.

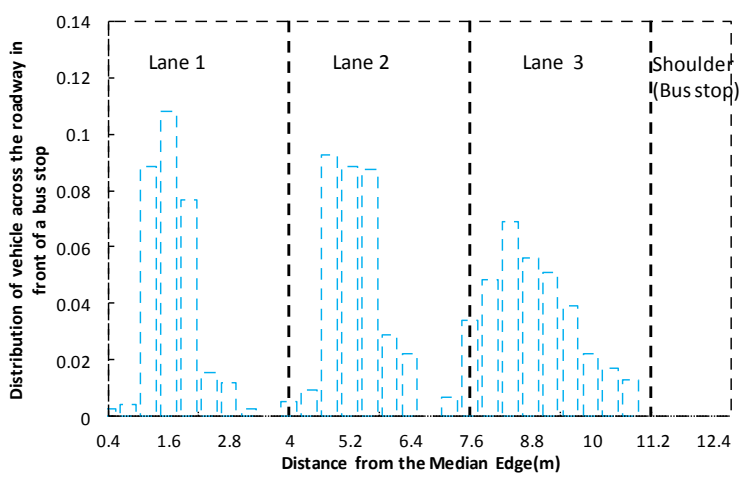

Fig.3 Distribution of distance from median edge

\section{Lateral offset}

Fig.1 displays the sample statistics that dispersion of the speed in each lane has some difference when the density level is the same.Since examination have shown that speed samples in every lane under every density level are following a normal distribution, this paper use the hypothesis testing to verify that there is significant difference among the average speed of three lanes when the density level is invariable. When $\mathrm{D}=1$, and lane 1 and lane 3 is chosen for example, the null hypothesis is

$$
H_{0}: \mu_{1} \neq \mu_{3}
$$

where, $\mu_{1}=$ the average speed in lane $1 ; \mu_{3}=$ the average speed in lane 3.

The test statistic is

$$
t=\frac{\bar{X}-\bar{Y}}{\sqrt{S_{1}^{2} / n_{1}+S_{3}^{2} / n_{3}}}
$$

where,

$\bar{X}, \bar{Y}=$ average speed the sample in lane 1 and lane 3;

$S_{1}^{2}, S_{3}^{2}=$ average speed variance of the two samples;

$n_{1}, n_{3}=$ sample sizes of the average speed.

Tab.2 shows the different t-test value that among three lanes under the same density level, where $\operatorname{sig}=$ the t-test value, and $v_{i j}=$ the average speed in lane $i$ when the density level is $j$.Take the significance level as 0.05 . If $s i g<0.05$, there is significant difference in the average speed, under the same density level,between the two lanes.
Table 2: Different T-Test Value

\begin{tabular}{cccccc}
\hline \hline$v_{i j}$ & sig & $v_{i j}$ & sig & $v_{i j}$ & sig \\
\hline$v_{11-} v_{21}$ & 0.011 & $v_{22}-v_{32}$ & 0.000 & $v_{14}-v_{34}$ & 0.868 \\
$v_{11}-v_{31}$ & 0.001 & $v_{13}-v_{23}$ & 0.108 & $v_{24}-v_{34}$ & 0.112 \\
$v_{21}-v_{31}$ & 0.000 & $v_{13}-v_{33}$ & 0.001 & $v_{15}-v_{25}$ & 0.074 \\
$v_{12}-v_{22}$ & 0.463 & $v_{23}-v_{33}$ & 0.036 & $v_{15}-v_{35}$ & 0.561 \\
$v_{12}-v_{32}$ & 0.000 & $v_{14-}-v_{24}$ & 0.105 & $v_{25}-v_{35}$ & 0.051 \\
\hline \hline
\end{tabular}

When $\mathrm{D}=1$, there is significant difference in the average speed among all three lanes. When $\mathrm{D}=2$ or $\mathrm{D}=3$, there is significant difference in the average speed only between lane 3 and the other two lanes, and there is no significant difference between lane 1 and lane 2 . When $\mathrm{D}=4$ or $\mathrm{D}=5$, there is no significant difference among three lanes at all.

As Fig.3 reveals that there is greatdifference in distribution of the lateral position among all three lanes, this paper supposes that the significant difference in the average speed is caused by different distribution of the lateral position. While the $\mathrm{D}=1$, vehicles are usually in a high speed. Once a vehicle is forced to make a lateral movement, it will slow down. When the lateral offset is large, the speed will down to a very low level, and the following vehicles and vehicles in the adjacent lane will also slow down a lot. Thus the average speed may be in any position with the change of the lateral offset. As lane 3 suffered the most severe lateral interference, its dispersion of the speed is the most in all three lanes. While $\mathrm{D}=2$ or $\mathrm{D}=3$, as there are several vehicles in the road, if the lateral offset is not so large, average speed won't reduce a lot. Therefore, vehicles in lane 3 will slow down a lot sometimes while vehicles in other two lanes may sometimes slow down a little. While $\mathrm{D}=4$ or $\mathrm{D}=5$, because there are already many vehicles on the road and average speed is already in a low level, so even the lateral offset is great, average speed won't reduce a lot.

In order to validate the hypothesis, which is different lateral position will affect the average speed, proposed in the previous part, this paper analyzed the relationship between lateral offset and the average speed in each lateral offset in lane 3 . As in Fig.4, When $\mathrm{D}=1$ and $\mathrm{D}=3$ are chosen for example. Average speed reduces with the increase of the lateral offset in the same density level, and average speed reduces with the increase of the density level when the lateral offset is the same as well. When $\mathrm{D}=3$, average speed reduces almost $40 \%$ when the lateral offset is increased from 0 to 9 , and as for $\mathrm{L}=0$, average speed reduces almost $46 \%$ when the density level is increased from 1 to 3. What's more, When $\mathrm{D}=4$ or $\mathrm{D}=5$, the lateral offsets of vehicles are rarely great. Because when there is a lateral movement occurred, it will create voids in front of the vehicle both in its own laneand the adjacent lane, and thus the road density level will decrease. However, the road speed will decline as the lateral movement often occurs with the brake. This indicates that though the decline of the speed is mainly caused by the increase of the road density, the lateral offset also affects the speed a lot. The same phenomena are also 
occurred in other density levels.

This paper also put the density level, average speed, and lateral offset together as in Fig.5. In the same density level, the low speed points are concentrating in the place where lateral offset is great. That is to say, if the discrete points in Fig.2 are stretched to the three-dimensional coordinates, with the same density level, the low speed point is mostly appearing with a great lateral offset, while the speed of the points with little lateral offset are mostly high. Therefore, the large dispersion of the speed in Fig. 2 is fundamentally caused by the great lateral offset. This also reveals that lateral offset does influent the average speed.

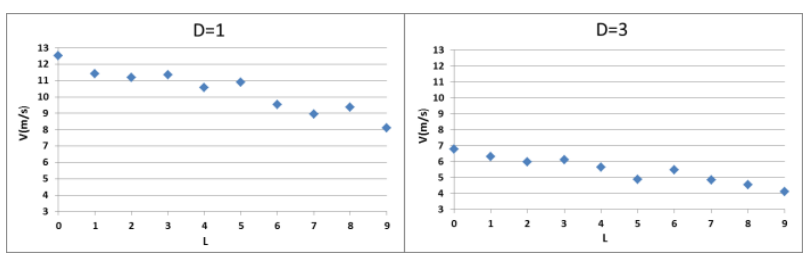

Fig.4 Figure of L-V

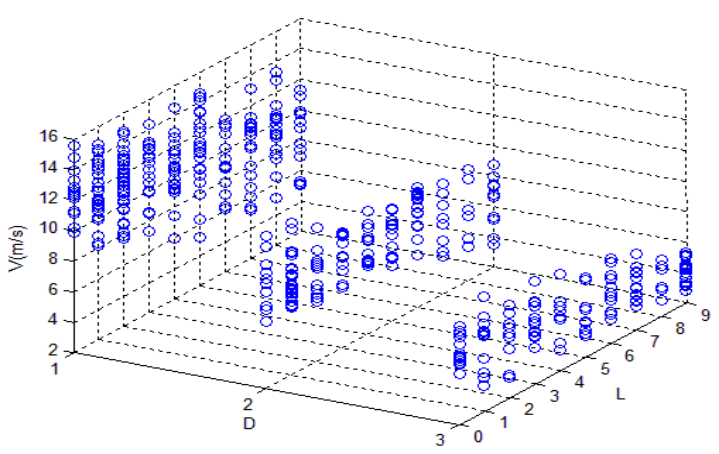

Fig.5 Figure of D-L-V

\section{Conclusion}

This paper focuses on the macroscopic traffic features like average speed and road density of urban bottleneck, and the lateral offset is also added to the analysis for intensively. The result shows that (1)there is much more lateral movement at traffic bottlenecks than ordinary sections; (2) when the density level is the same, average speed will decline with the increasing of the lateral offset; (3) road density may not so high when the lateral offset is great; (4) lateral offset does affect the road speed a lotthough the decline of the speed is mainly caused by the increasing of the road density. Because there is huge number of lateral movement at the traffic bottlenecks, not only the average speed is lower than the speed in other sections, but the actual capacity is also lower than the design capacity. So it is easy to going into a congested state in the traffic bottlenecks as well. What's more,the real situation on the road is: in many times though the road density is not so high, the average speed of the road is still low, and sometimes even come into a traffic jam. This is what macro fundamental diagram can't describe, and the next work after this paper is to analyze this phenomena by microscopic traffic flow model which is more subtly in describing the behavior of the vehicles.

\section{Acknowledgment}

This paper is supported by National High Technology Research and Development Program of China (863 Program). (No.2011AA110306)

\section{References}

[1] Makoto Nakata, Atsuo Yamauchi, Jun Tanimoto, AyaHagishima, Dilemma game structure hidden in traffic flow at a bottleneck due to a 2 into 1 lane junction[J]. Physica A, 2010, 5353-5361.

[2] Carlos F. Daganzo, Jorge A. Laval, Moving bottlenecks: A numerical method that converges in flows[J]. Transportation Research Part B, 2005, 39: 855-863.

[3] Carlos F. Daganzo, Michael J. Cassidy, Effects of high occupancy vehicle lanes on freeway congestion [J]. Transportation Research Part B, 2008, 42: 861-872.

[4] Michael J.Cassidy, RobertL.Bertini,Some traffic features at freeway bottlenecks[J].Transportation Research Part B, 1999, 33: 25-42.

[5] GONG L J, YANG D Y, Analysis of Road Traffic Bottleneck Based on Loop Detector Data[J]. Journal of Highway and Transportation Research and Development, 2010, 27(7): 1002-0268.

[6] GUO Zhong-hua, WANG Wei, LU Jian, Analyssis of Road Traffic Flow Affected by Bus Stops Without Bus Bay[J]. Journal of Highway and Transportation Research and Development, 2005, 11: 138-143

[7] SHAO Chang-qiao, ZHANG Zhi-yong, RongJian,Analysis of Flow Characteristics at Freeway Bottlenecks[J]. Journal of Beijing University of Technology, 2009, 3:354-358.

[8] BanihanGunay, Car following theory with lateral discomfort[J].Transportation Research Part B, 2007, 41: 722-735.

[9] Alfonso Montella, Massimo Aria, Antonio D'Ambrosio, Francesco Galante, FilomenaMauriello, Mariano Pernetti, Simulator evaluation of divers' speed, deceleration and lateral position at rural intersections in relation to different perceptual cues[J].Accident Analysis and Prevention, 2011, 2072-2084.

[10] Xiaojian $\mathrm{Hu}$, Wei Wang, Haifei Yang, Mixed traffic flow model considering illegal lane-changing behavior: Simulations in the framework of Kerner's three-phase theory[J].Physica A, 2012, 5102-5111. 